\title{
Optimization of Streptomyces bacteriophage $\phi$ C31 integrase system to prevent post integrative gene silencing in pulmonary type II cells
}

\author{
Manish Kumar Aneja ${ }^{1}$, Johannes Geiger ${ }^{1,2}$, \\ Rabea Imker ${ }^{1}$, Senta Üzgun ${ }^{1,2}$, \\ Michael Kormann ${ }^{1}$, Guenther Hasenpusch ${ }^{1}$, \\ Christof Maucksch ${ }^{1,2}$ and Carsten Rudolph ${ }^{1,2,3}$ \\ ${ }^{1}$ Division of Molecular Pulmonology \\ Department of Pediatrics \\ Ludwig-Maximilians University \\ Lindwurmstrasse $2 \mathrm{~A}$ \\ 80337 Munich, Germany \\ ${ }^{2}$ Department of Pharmacy \\ Free University of Berlin \\ Takustrasse 3 \\ 14166 Berlin, Germany \\ ${ }^{3}$ Corresponding author: Tel, 49-8951607711; Fax, 49-8951604421; \\ E-mail, Carsten.Rudolph@med.uni-muenchen.de \\ DOI 10.3858/emm.2009.41.12.098
}

Accepted 14 August 2009

Abbreviations: AZA, 5-aza-2'deoxycytidine; EF1 $\alpha$, elongation factor $1 \alpha$; Int-NLS, integrase with a C-terminal nuclear localization signal; L-PEI, linear polyethylenimine; TSA, trichostatin A; UBB, ubiquitin $\mathrm{B}$; UBC, ubiquitin $\mathrm{C}$

\begin{abstract}
$\phi C 31$ integrase has emerged as a potent tool for achieving long-term gene expression in different tissues. The present study aimed at optimizing elements of $\phi C 31$ integrase system for alveolar type II cells. Luciferase and $\beta$-galactosidase activities were measured at different time points post transfection. 5-Aza-2'deoxycytidine (AZA) and trichostatin A (TSA) were used to inhibit DNA methyltransferase and histone deacetylase complex (HDAC) respectively. In A549 cells, expression of the integrase using a CMV promoter resulted in highest integrase activity, whereas in MLE12 cells, both CAG and CMV promoter were equally effective. Effect of polyA site was observed only in A549 cells, where replacement of SV40 polyA by bovine growth hormone (BGH) polyA site resulted in an enhancement of integrase activity. Addition of a C-terminal SV40 nuclear localization signal (NLS) did not result in any significant increase in integrase activity. Long-term expression
\end{abstract}

studies with AZA and TSA, provided evidence for post-integrative gene silencing. In MLE12 cells, both DNA methylases and HDACs played a significant role in silencing, whereas in A549 cells, it could be attributed majorly to HDAC activity. Donor plasmids comprising cellular promoters ubiquitin $B$ (UBB), ubiquitin $C$ (UCC) and elongation factor $1 \alpha(E F 1 \alpha)$ in an improved backbone prevented post-integrative gene silencing. In contrast to A549 and MLE12 cells, no silencing could be observed in human bronchial epithelial cells, BEAS-2B. Donor plasmid coding for murine erythropoietin under the EF1 $\alpha$ promoter when combined with $\phi$ C31 integrase resulted in higher long-term erythropoietin expression and subsequently higher hematocrit levels in mice after intravenous delivery to the lungs. These results provide evidence for cell specific post integrative gene silencing with $\phi \mathrm{C} 31$ integrase and demonstrate the pivotal role of donor plasmid in long-term expression attained with this system.

Keywords: gene silencing; gene therapy; integrases; lung; plasmids; promoter regions, genetic

\section{Introduction}

A variety of viral and nonviral gene vectors have been evaluated in numerous pulmonary gene therapy approaches. Currently most gene therapy applications use replication-deficient viruses as gene transfer agents to deliver therapeutic DNA into cellular targets. While viral vectors are effective in vivo, they also have numerous disadvantages including induction of immune response and eventually insertional mutagenesis (Lee et al., 2005; Davis and Cooper, 2007).

The efficacy of nonviral vectors in correcting genetic diseases has been hampered by poor delivery to target cells, lack of integration and long-term gene expression. Recently, plasmid based nonviral gene transfer systems capable of mediating stable genomic integration of transgenes have been described. One such system is the integrase from Streptomyces phage $\phi \mathrm{C} 31$. It was demonstrated that $\phi \mathrm{C} 31$ integrase mediates site-specific integration into the host genome of mammalian cells in vitro and in vivo by recombining the attB 
recognition site in an episomal plasmid and one or more pseudo-attP sites in the host chromosomes (Thyagarajan et al., 2001; Olivares et al., 2002). $\phi$ C31 integrase has been used for gene therapy approaches and was tested in vitro and in vivo in mice (Olivares et al., 2002; Ortiz-Urda et al., 2002; Held et al., 2005; Bertoni et al., 2006). Recently, the activity of this enzyme system has been demonstrated in murine lungs (Aneja et al., 2007). Long-term expression of luciferase, was achieved by co-delivery of an integrase expression plasmid and was associated with the genomic integration of the transgene. However, the final stable long-term expression values obtained in the above mentioned study were $\sim 1000$-fold lower compared to values observed immediately after gene delivery.

The present study was aimed at optimizing the elements of $\phi C 31$ integrase system, primarily the integrase expression construct and the donor plasmid (plasmid to be integrated). Each of these parameters was independently investigated with respect to its effect on $\phi \mathrm{C} 31$ integrase mediated recombination and long-term expression. To optimize the integrase expression construct for alveolar type II cells, effect of different promoters, poly A sites and a C-terminal NLS on the activity of $\phi$ C31 integrase were investigated. Using an intramolecular recombination assay described earlier (Aneja et al., 2007; Maucksch et al., 2008), we demonstrate that both promoter and polyA sequence elements influence the acitivty of $\phi C 31$ integrase.

In previous studies, nuclear localization signal (NLS) of the SV40 large $T$ antigen, has been added at both the $\mathrm{N}$ and C-terminus of $\phi \mathrm{C} 31$ integrase (Andreas et al., 2002; Raymond and Soriano, 2007). Using an extrachromosomal plasmid carrying attB and attP sites, that must be recombined to express the reporter protein, it was reported that the $\phi \mathrm{C} 31$ integrase carrying a C-terminal NLS performed intramolecular excision 1.7-fold better than the wild-type enzyme (Andreas et al., 2002). When the same reporter plasmid was integrated into the chromosomes of NIH3T3 cells, expression of the reporter protein was 10-fold higher in cells that received the $\phi C 31$ integrase with NLS compared to the wildtype (Andreas et al., 2002). In another study, $\phi C 31$ integrase gene was codon optimized for expression in mouse and simultaneously modified to carry a C-terminal NLS (Raymond and Soriano, 2007). However, for integration of plasmid DNA into the genome, the reaction of interest in gene therapy, $\phi$ C31 integrase with NLS has not been compared to the wild-type $\phi$ C31 integrase in either tissue culture cells or in an in vivo gene therapy setting. In the present study, we directly compared the integration of donor plasmid and subsequent long-term expression, when expression plasmids coding for an integrase with or without a C-terminal NLS were co-delivered.

In subsequent experiments, the donor plasmid was optimized for supporting long-term expression when integrated with the $\phi \mathrm{C} 31$ integrase. Previous studies using non-integrated plasmids, have shown that the choice of promoter significantly influences the long-term expression of the transgene. Relatively long-term expression (compared to viral promoters) has been achieved using a hybrid ubiquitin B (UBB, Yew et al., 2001), ubiquitin C (UBC) and elongation factor $1 \alpha(E F 1 \alpha)$ promoters (Gill et al., 2001). Though the expression from these cellular promoters was detected longer than the viral promoters like CMV or RSV, there was a decline in expression over time, most probably due to the loss of the plasmid DNA during cell turnover. Integration into the chromosomal DNA would prevent the loss of the plasmid during cell division but would then place these promoters in a different chromatin environment compared to their episomal state. In the present study, we analysed the expression kinetics from these promoters, when integrated by $\phi C 31$ integrase.

Another cause for the loss of transgene expression has been the presence of $\mathrm{CpG}$ motifs and bacterial backbone sequences present in plasmid DNA preparations. Recent studies have shown that depletion of $\mathrm{CpG}$ sequences in plasmid vectors can reduce the silencing effects and prolong gene expression (Yew et al., 2002; Chen et al., 2003). In the present study, different promoters were used to drive the expression of luciferase in the donor plasmid backbone described earlier (Yew et al., 2001) and these new donor plasmids were then screened for post-integrative gene silencing. The plasmid backbone pGZCUBI (Yew et al., 2001) contains an unmodified ubiquitin $B$ promoter and intron, but a $\mathrm{CpG}$ reduced backbone composed of a synthetic, non-CpG gene conferring resistance to kanamycin and a minimal replication origin. Using DNA methylase and histone deacetylation (HDAC) inhibitors (5-aza-2'deoxycytidine and trichostatin $A$, respectively), it could be shown that the extent of post-integrative gene silencing varied based on the bacterial backbone of the plasmid vector and the promoter used. The effect of each of these parameters was observed to be cell type and species specific.

Since the donor plasmid with EF1 $\alpha$ promoter in pGZCUBI backbone was not silenced in both A549 and MLE12 cells, the efficiency of this plasmid backbone to support long-term expression resulting in a physiological response, when combined with 
$\phi$ C31 integrase, was analysed in vivo in mice. For this, murine erythropoietin was used as a physiological target. The main physiological effect of erythropoietin is on erythropoiesis which can be evaluated through hematocrit measurement. This provided an easy readout in mice experiments. For gene delivery to the lungs, pDNA was complexed with the polycation polyethylenimine (PEI) to compact the plasmid molecule and to protect it from degradation (Demeneix and Behr, 2005). PEI has been shown to be a powerful agent for transfecting the mouse lung after injection of plasmid-based polyplexes through the tail vein. It has been shown that most cell types in the lung are transfected including type I and type II pneumocytes (Goula et al., 2000; Dif et al., 2006). Long-term expression of erythropoietin and resulting higher hematocrit values were observed after co-delivery of an integrase expression plasmid.

The present report suggests that post-integrative gene silencing may be an under-appreciated obstacle to $\phi \mathrm{C} 31$ integrase-based gene therapy approaches. The results presented here clearly reveal that the design of the donor plasmid plays a pivotal role in determining the stable long-term expression values attained with $\phi \mathrm{C} 31$ integrase. Moreover, post-integrative gene silencing with different promoters and plasmid backbone appear to be cell specific. This optimized $\phi C 31$ integrase system, may be potentially applicable to gene therapy of a variety of lung diseases such as cystic fibrosis and especially secreted proteins like $\alpha 1$-antitrypsin deficiency.

A

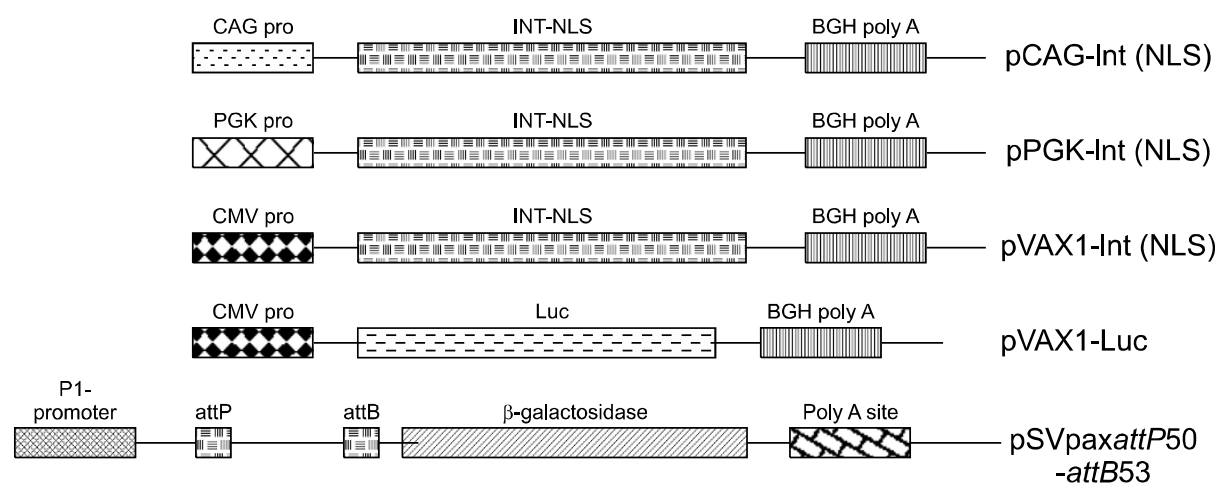

B

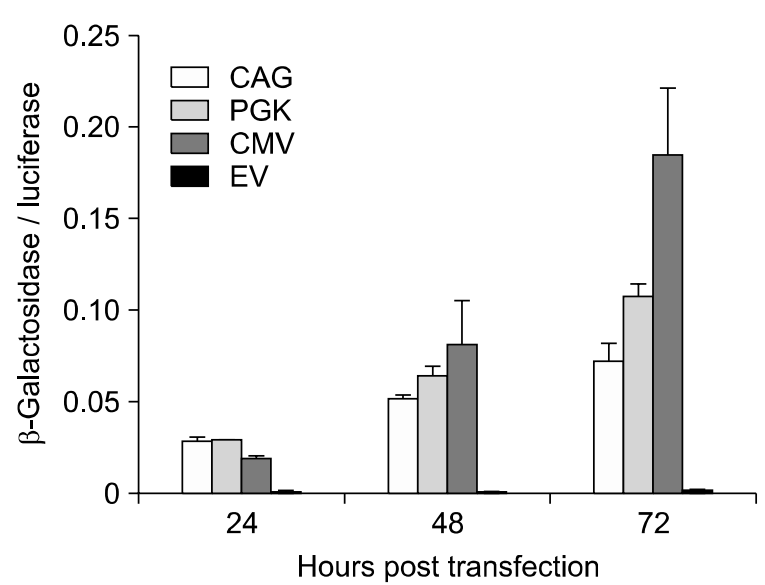

C

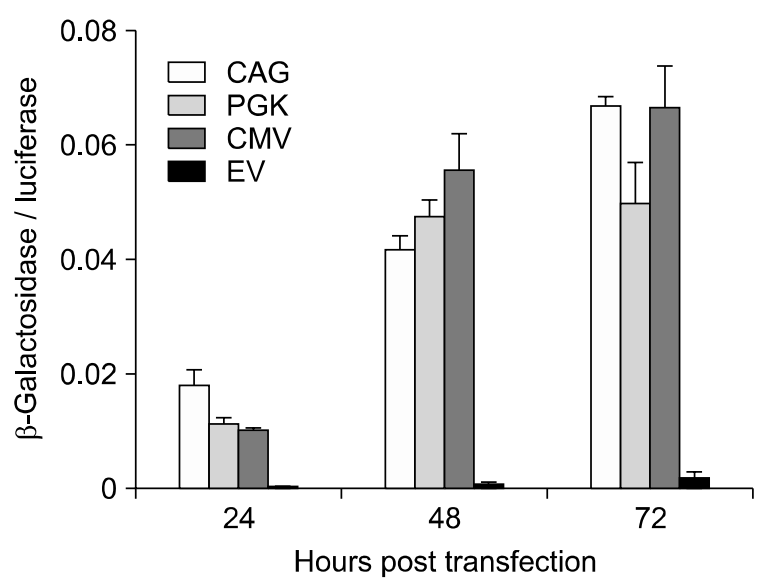

Figure 1. (A) Schematic representation of the different integrase expression and the reporter plasmids used to investigate the effect of various promoters on the activity of $\phi C 31$ integrase in A549 (B) and MLE-12 (C) cells. Cells were transfected with 100 ng of pSVpaxattP50-attB53 + 50 ng of pVAX1-luc and equal number of molecules of the different integrase encoding plasmids. pVAX1 was used as vector control and also filler DNA to make the total amount of DNA (800 ng) equal in all the compared samples. $\beta$-galactosidase, luciferase and total protein were measured at 24,48 and $72 \mathrm{~h}$ post transfection. Each transfection was repeated twice with three independent replicates per experiment. Values represent mean \pm standard deviation $(n=6)$ of the ratio of $\beta$-galactosidase to luciferase. EV. Vector control 


\section{Results}

\section{Optimization of the $\phi \mathrm{C} 31$ integrase expression plasmid}

Elements of the expression cassette greatly influence the kinetics of transgene expression. Our previous results in Jurkat cells with the integrase mRNA (Maucksch et al., 2008) provide initial evidence that the kinetics of integrase expression may play an important role in achieving higher long-term expression with $\phi \mathrm{C} 31$ integrase. To address this aspect, effect of different promoter and polyA sequences in the integrase expression plasmid on the enzymes recombinational activity was investigated into. For this, an intramolecular recombination assay based on the measurement of $\beta$-galactosidase activity, as an indirect indicator of integrase activity, was used. In the construct pSVpaxattP50-attB53, recombination between wild type attP and attB sequences brought $\beta$-galactosidase CDNA in frame with the promoter. In the absence of integrase, no $\beta$-galactosidase activity above background could be detected. Luciferase values were used to normalize the transfection efficiencies. To measure the effect of different promoters, the reporter plasmid pSVpaxattP50attB53 was co-delivered with equal number of plasmid copies of pCAG-Int (NLS), pPGK-Int (NLS), pVAX1-Int (NLS) or pVAX1 together with 50 ng of pVAX1-luc (Figure 1A). Measurement of luciferase and $\beta$-galactosidase was performed at 24, 48 and $72 \mathrm{~h}$ post transfection. In A549 cells (Figure 1B), expression from CMV promoter resulted in 2.5-fold higher integrase activity than from the CAG promoter $(P \leq 0.01)$ and 1.8 -fold higher compared to the PGK promoter $(P \leq 0.01)$ at $72 \mathrm{~h}$ post transfection. In MLE12 cells (Figure 1C), expression from PGK promoter resulted in lowest activity compared to CAG (1.3 fold: $P \leq$

A

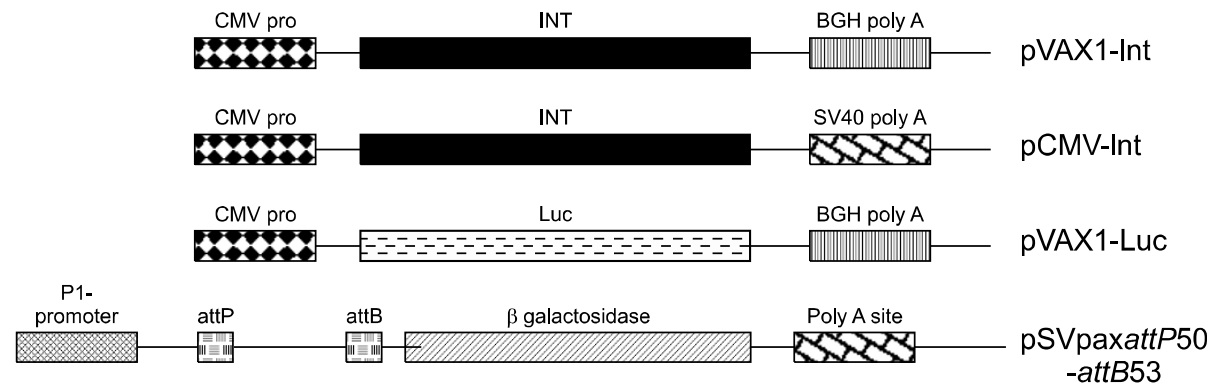

B

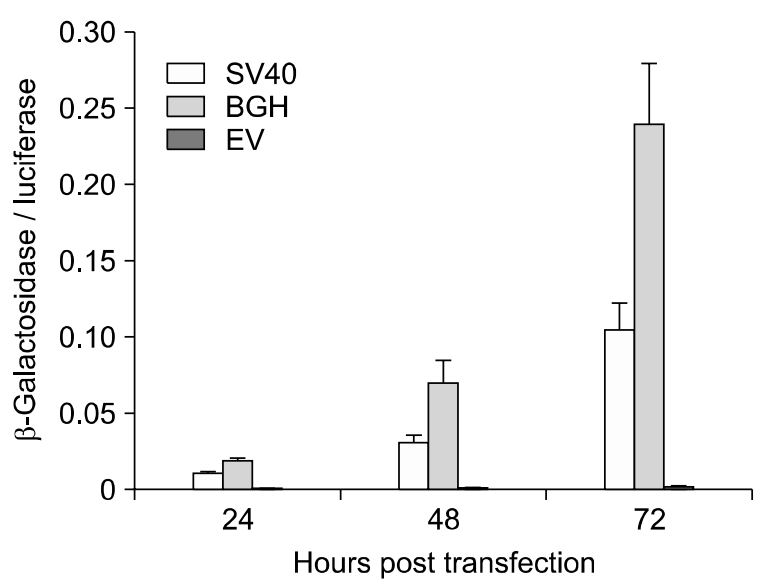

C

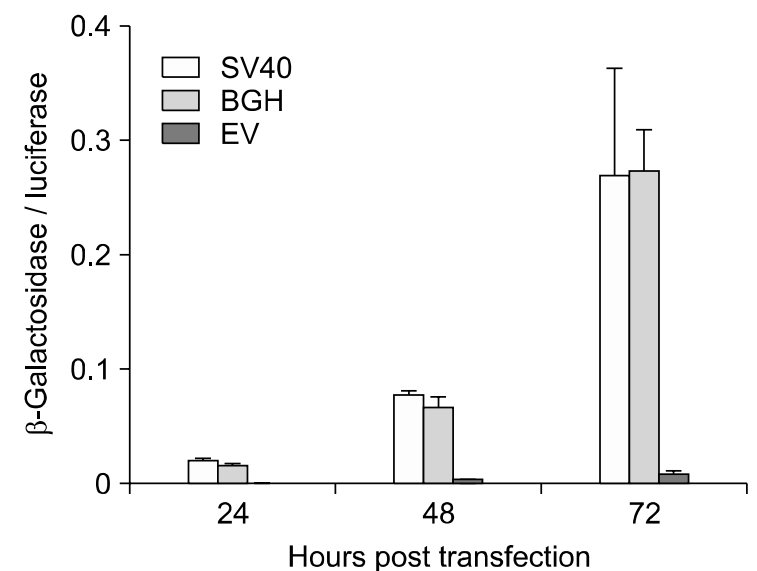

Figure 2. (A) Schematic representation of the different integrase expression and the reporter plasmids used to investigate the effect of poly $A$ sites on the activity of $\phi C 31$ integrase in A549 (B) and MLE-12 (C) cells. Cells were transfected with $100 \mathrm{ng}$ of pSVpaxattP50-attB53 + 50 ng of pVAX1-luc and equal number of molecules of the different integrase encoding plasmids. pVAX1 was used as vector control and also filler DNA to make the total amount of DNA $(800 \mathrm{ng})$ equal in all the compared samples. $\beta$-galactosidase, luciferase and total protein were measured at 24,48 and $72 \mathrm{~h}$ post transfection. Each transfection was repeated twice with three independent replicates per experiment. Values represent mean \pm standard deviation $(n=6)$ of the ratio of $\beta$-galactosidase to luciferase. EV. Vector control. 
$0.02)$ and CMV promoter $(P \leq 0.05)$ at $72 \mathrm{~h}$ post transfection.

Another feature of the expression cassette that influences transgene expression is the polyadenylation signal. In the present study, the SV40 polyA site was compared to that of the bovine growth hormone $(\mathrm{BGH})$. Reporter plasmid pSVpaxattP50attB53 was co-delivered with equal number of plasmid copies of pCMV-Int, pVAX1-Int or pVAX1 together with $50 \mathrm{ng}$ of pVAX1-luc (Figure 2A). Like the assay for the comparison of different promoters, luciferase and $\beta$-galactosidase activities were

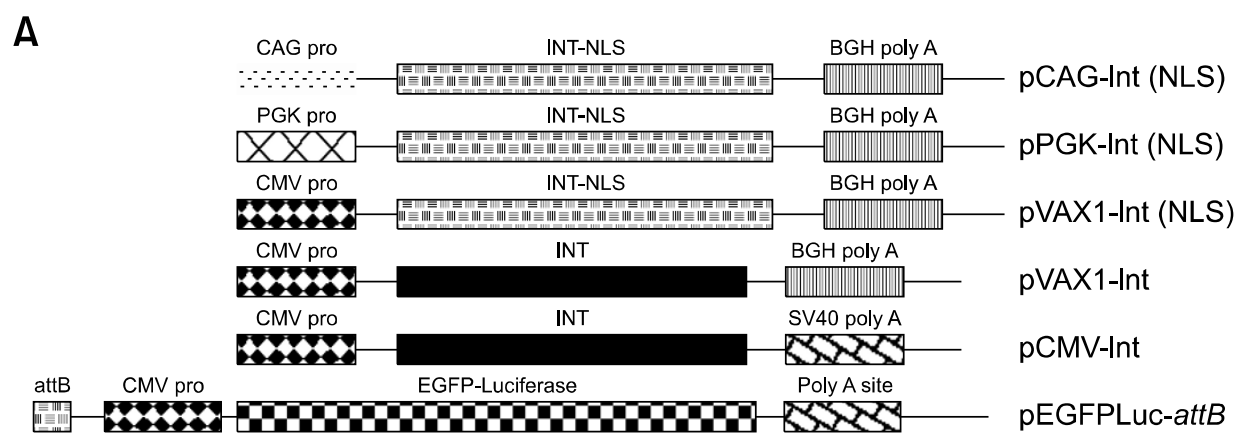

B

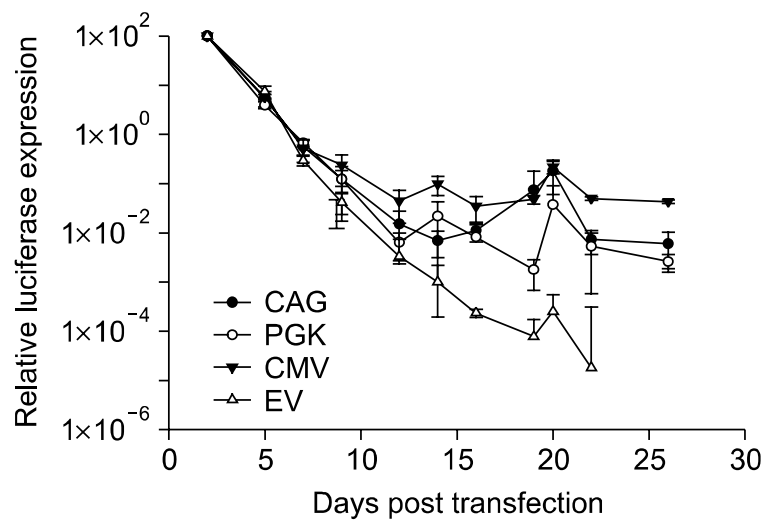

C

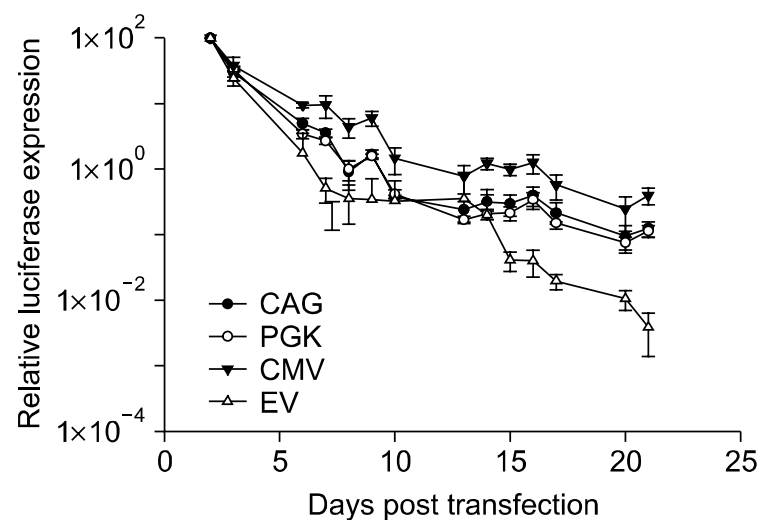

D

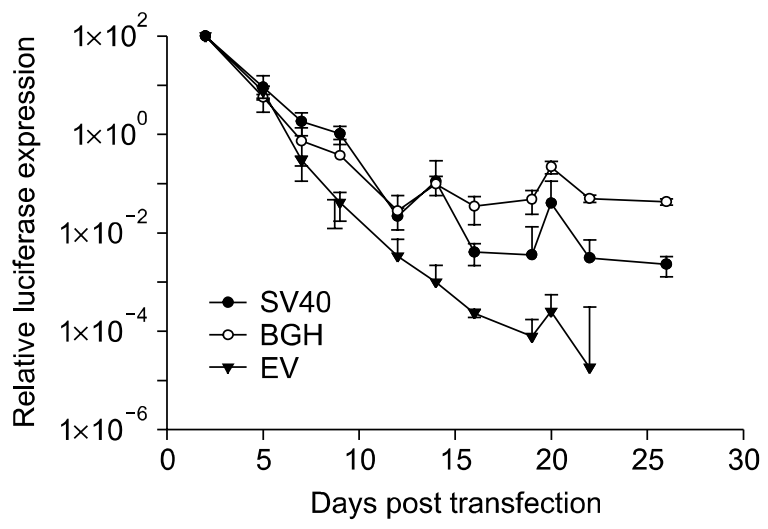

$E$

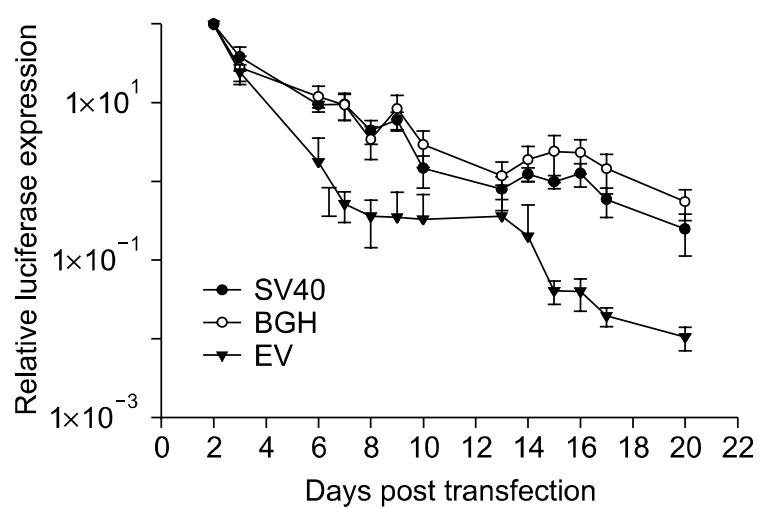

Figure 3. (A) Schematic representation of the different integrase expression and the donor plasmids used to in the subsequent long-term expression assays. Effect of different promoters and poly A sites on $\phi C 31$ integrase mediated long-term expression in A549 (B, D) and MLE-12 (C, E) cells. Cells were transfected with $200 \mathrm{ng}$ of pEGFPLucattB and equal number of molecules of the different integrase encoding plasmids. pVAX1 was used as vector control and also filler DNA to make the total amount of DNA ( $800 \mathrm{ng}$ ) equal in all the compared samples. Luciferase and total protein were measured at different time points. Long-term expression results are expressed as percentage of luciferase values two days after transfection. Each transfection was repeated twice with two independent replicates per experiment. Values represent mean \pm standard deviation $(n=4)$. EV. Vector control. 
measured 24, 48 and $72 \mathrm{~h}$ post transfection. In human A549 cells (Figure 2B), BGH polyA site resulted in 2.3-fold higher integrase activity compared to SV40 polyA site $(P \leq 0.01)$ after $72 \mathrm{~h}$. However, in MLE12 cells (Figure 2C), no effect of poly A site could be observed at any of the measured time points.

Since kinetics and efficiencies of intra- and inter-molecular recombination are quite different, we also investigated the effect of different promoters and polyA sites on integrase mediated long-term expression. Plasmid constructs used in these sets of experiments have been schematically presented as Figure 3A. In accordance with our intra-molecular recombination assay results, expression of $\phi C 31$ integrase from the CMV promoter (Figure $3 \mathrm{~B}$ ) and the presence of a BGH polyA site (Figure 3D) resulted in significantly $(P \leq 0.05)$ higher long-term expression in A549 cells. In MLE12 cells, the difference between different promoters was not as strong as in A549 cells (Figure 3C) and no effect of polyA site on long-term expression values could be observed (Figure $3 \mathrm{E}$ ). These results with the intra-molecular recombination assay and the long-term expression data, provide evidence that the design of the integrase expression construct influences its activity in alveolar cells.

\section{Effect of C-terminal NLS on the activity of $\phi$ C31 integrase with respect to long-term expression}

Addition of a C-terminal NLS has been shown to enhance the efficiency of $\phi C 31$ integrase (Andreas et al., 2002; Raymond and Soriano, 2007) presumably by facilitating enhanced nuclear transport of the enzyme. MLE12 cells were co-transfected with pEGFPLucattB and pVAX1-Int (NLS), pVAX1-Int (Figure 4A) or pVAX1, respectively. Luciferase activity was measured at different time points. Co-transfection of the integrase expression plasmid resulted in long-term expression of luciferase over a three-week time period (Figure 4B). However, there was no significant difference between the long-term expression values achieved with either pVAX1-Int (NLS) or pVAX1-Int. At 22 days post transfection, total DNA was isolated from the cells and the amount of pEGFPLucattB was quantified by real time PCR. In accordance with the long-term expression data, the differences between either of the integrase type and vector control were signi-

A

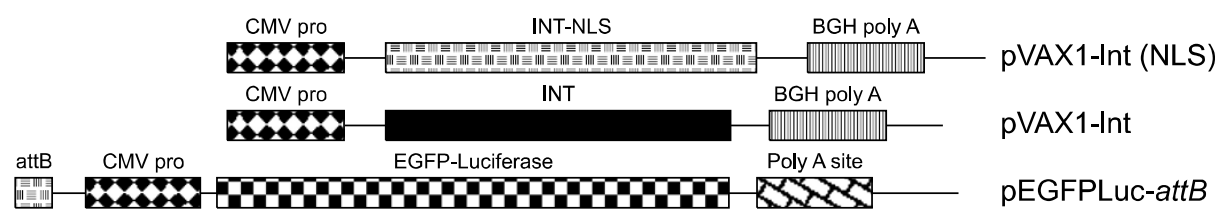

B

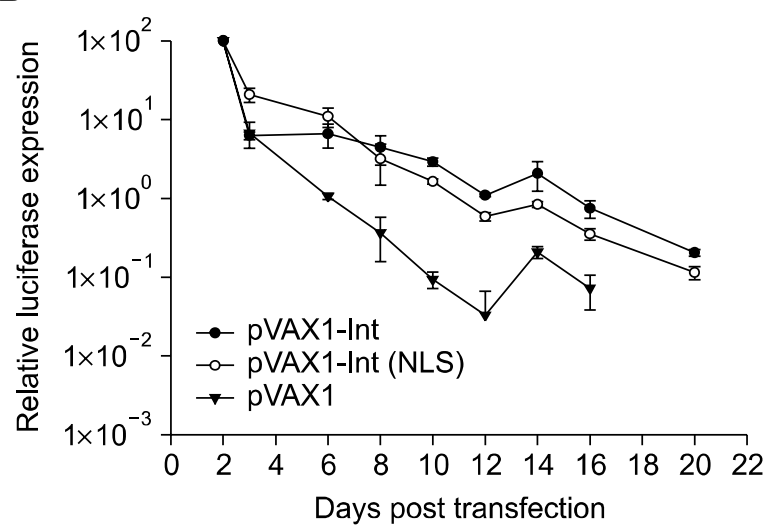

C

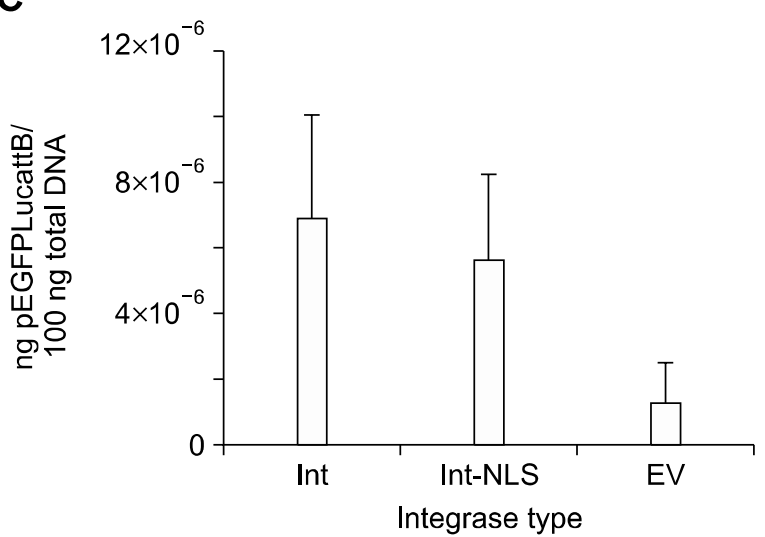

Figure 4. Effect of C-terminal NLS on $\phi C 31$ integrase mediated long-term expression in MLE12 cells. (A) Schematic representation of the different integrase expression and the donor plasmids used in this assay. (B) Cells were transfected with $200 \mathrm{ng}$ of pEGFPLuc-attB + equal number of molecules of the different integrase encoding plasmids. Luciferase activity was measured at different time points. Long-term expression results are expressed as percentage of luciferase values two days after transfection. Values represent mean \pm standard deviation $(n=6)$. (C) Quantification of pEGFPLucattB by real time PCR. At 22 days post transfection described in Figure 4B, total DNA was isolated from the cells and the amount of pEGFPLucattB quantified. Each transfection was repeated twice with three independent replicates per experiment. Values represent the mean \pm standard deviation $(n=6)$. Int: integrase; Int-NLS: integrase with C-terminal NLS. 
ficant $(P \leq 0.02)$. There was no significant difference between the total amount of pEGFPLucattB detected when either of the integrase expressing constructs (with and without NLS) were co-delivered (Figure 4C). Similar results were obtained in A549 cells (data not shown).

\section{Optimization of the donor plasmid}

Though the use of CMV promoter and BGH polyA site resulted in an increase in the long-term expression values in A549 cells, none of these could prevent the steady fall in long-term expression values (Figure 3 ). We next investigated the

\section{A}

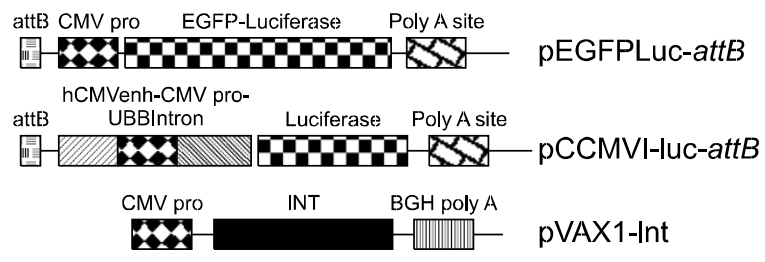

B

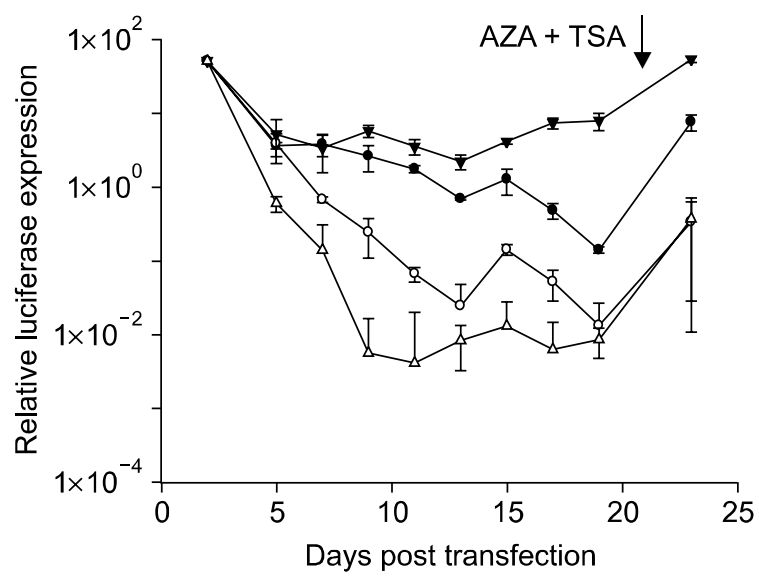

role of the donor plasmid in this observed steady decline in expression. For these experiments, pVAX1-Int was used as the integrase expression plasmid based on the favourable features of CMV promoter and $\mathrm{BGH}$ polyA site, which resulted in highest integrase activity as measured by the intramolecular recombination and long-term expression assays.

Silencing of the transgene post integratively has been described recently for the Sleeping Beauty transposon system. Both DNA methylases and histone deacytylase complex (HDAC) have been proposed to be involved in the mechanism (Garrison et al., 2007). Plasmid constructs used in
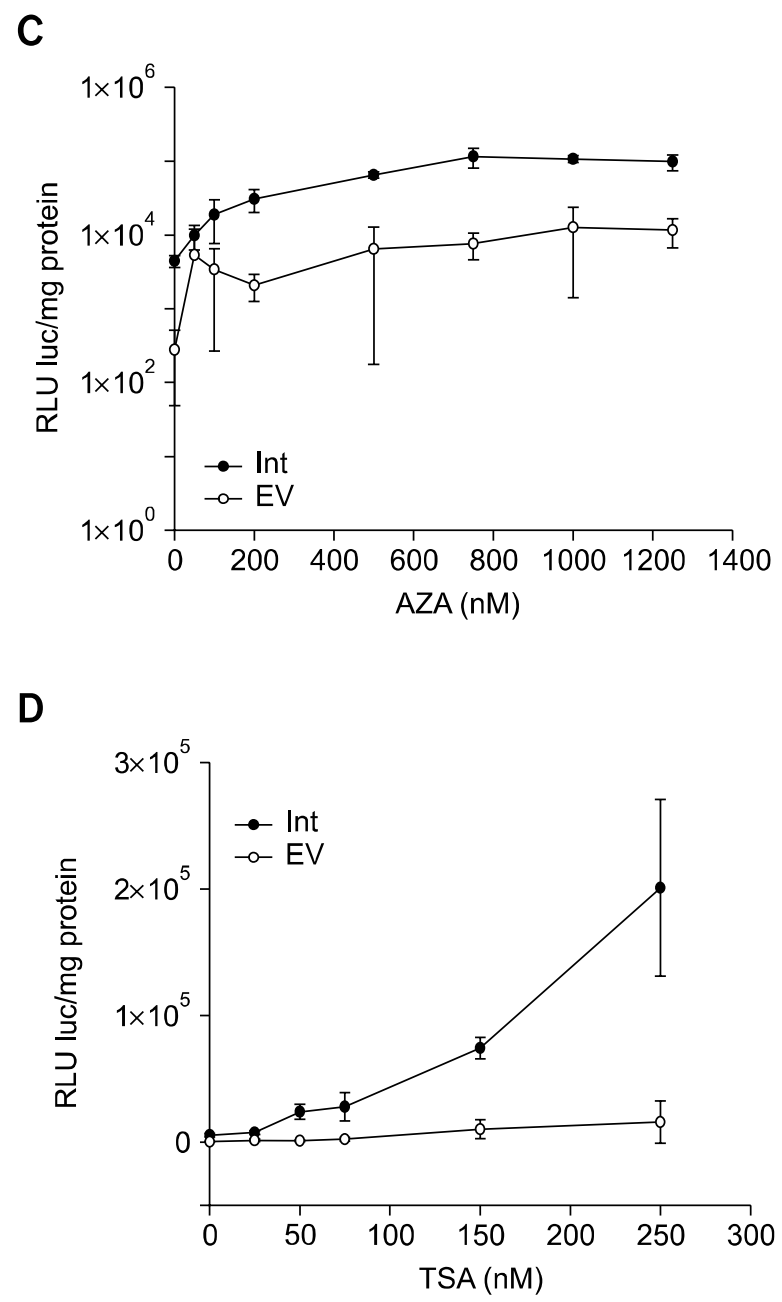

Figure 5. Gene silencing after integration with $\phi \mathrm{C} 31$ integrase is bacterial backbone dependent in MLE12 cells. (A) Schematic representation of the plasmid DNA constructs used in this set of experiments. (B) MLE12 cells were transfected either with pEGFPLucattB \pm pVAX1-Int or pCCMVI-luc-attB \pm pVAX1-Int. AZA and TSA were added at 21 days post transfection. Luciferase activity was measured at different time points. Long-term expression results are expressed as percentage of luciferase values two days after transfection. (C) MLE12 cells were transfected with pEGFPLucattB \pm pVAX1-Int. Increasing concentrations of AZA were added at 21 days post transfection and luciferase measurements made $48 \mathrm{~h}$ subsequently. (D) MLE12 cells were transfected with pEGFPLucattB \pm pVAX1-Int. Increasing concentrations of TSA were added at 21 days post transfection and luciferase measurements made $48 \mathrm{~h}$ subsequently. Each transfection was repeated twice with two independent replicates per experiment. Values represent mean \pm standard deviation $(n=4)$. Int: Integrase construct; EV: pVAX1 used as vector control. 
the subsequent experiments are presented as Figure 5A. DNA methylase and HDAC inhibitors: $A Z A$ and TSA respectively, were added to MLE12 cells co-transfected with pEGFPLucattB and pVAX1-Int or pVAX1 at 21 days post transfection. At this time point, only residual luciferase expression could be detected in the vector control group. Luciferase values were measured $48 \mathrm{~h}$ subsequent to treatment and a highly significant $(P$ $\leq 0.01$ ), 50-fold increase in expression could be observed (Figure 5B). A similar increase was also observed in the control group without integrase. This experiment provided us the evidence that the plasmid pEGFPLucattB was slowly silenced over time whether integrated or not.

To further dissect out the role of DNA methylases and HDACs, increasing amounts of AZA or TSA were added separately and luciferase values were measured $48 \mathrm{~h}$ subsequently. Inhibition of DNA methylases by $A Z A$, resulted in 22-fold higher luciferase expression in the integrase group where as the expression in the control group went up approximately 40-fold (Figure 5C). In contrast, when HDACs were inhibited by TSA, a significant 37-fold increase in expression was observed only in the integrase group. No significant increase in expression could be observed in the control group (Figure 5D). These data demonstrate that both DNA methylases and HDACs play significant roles in silencing plasmids integrated with $\phi$ C31 integrase.

Since both vector backbone and promoters are reported to play a role in gene silencing, we further addressed the role of each of these elements. As a control for the pEGFPLucattB backbone, a previously described plasmid backbone pGZCUBI (Yew et al., 2001), was used. Luciferase cDNA, CMV promoter and the attB binding site were cloned into this vector to generate pCCMVI-luc-attB (Figure $5 A)$. Long-term expression experiment similar to that performed with $p E G F P L u c a t t B$ revealed that plasmid backbone plays a pivotal role in post integrative gene silencing. The steady decline in expression as observed with pEGFPLucattB was not evident with the improved donor plasmid which contained the same CMV promoter as in pEGFPLucattB (Figure 5B). The stable long-term expression values observed with this improved donor plasmid was approximately 10-fold lower when compared to the values observed at day 2 post transfection, unlike with pEGFPLucattB where the stable expression values were approximately 1000 -fold lower. Addition of AZA and TSA at 21 days post transfection, revealed a less extensive silencing of the plasmid when integrated with $\phi \mathrm{C} 31$ integrase $(\sim 10$-fold $)$ compared to the control group ( $\sim 50$ fold). These results in MLE12 cells provide evidence for the role of bacterial backbone sequences in silencing transgenes integrated with $\phi$ C31 integrase.

In contrast to the observations in MLE12 cells, no effect of bacterial backbone in post integrative gene silencing could be observed in human A549 cells. Measurement of luciferase expression at different time-points revealed similar kinetics of luciferase expression from the CMV promoter in pEGFPLucattB and pCCMVI-luc-attB (Figure 6A). The expression declined steadily before it stabilized at levels approximately 500-fold lower than the values observed at day 2 post transfection. Addition of AZA and TSA at 26 days post transfection did not result in any drastic increase in gene expression $48 \mathrm{~h}$ subsequently. However, like in MLE12 cells, the construct pCCMVI-luc-attB when integrated with $\phi C 31$ integrase was less extensively silenced compared to the control group.

Similar to the experiments done in MLE12 cells aiming to dissect out the role of DNA methylases and HDACs, increasing amounts of AZA or TSA were added separately and luciferase values were measured $48 \mathrm{~h}$ subsequently. In contrast to the results in MLE12 cells, where inhibition of DNA methylases resulted in a significant 22-fold higher luciferase expression in the integrase and 40-fold higher in the control groups respectively, no such increase was observed in A549 cells (Figure 6B). Thus DNA methylases may not play a significant role in post-integrative gene silencing in human alveolar type II cells. Inhibition of HDACs by TSA, resulted in a significant 15 -fold increase in luciferase expression only in the integrase group (Figure 6C). This is similar to observations made in MLE12 cells. These data demonstrate that in human A549 cells, silencing of plasmids integrated by $\phi$ C 31 integrase, is mediated majorly by HDACs, unlike in MLE12 cells, where both DNA methylases and HDACs play significant roles.

In an attempt to prevent this post integrative gene silencing, donor plasmids comprising cellular promoters, ubiquitin B (UBB), ubiquitin C (UBC) and elongation factor $1 \alpha(E F 1 \alpha)$ were constructed resulting in pCUBI-luc-att $B, p C U C l-l u c-a t t B$ and pCEF1 $\alpha$ l-luc-attB, respectively (Figure 7A). The three constructs (with UBB, UBC and EF $1 \alpha$ promoters, respectively) were then compared in the long-term expression assay. MLE12 and A549 cells were co-transfected with pVAX1-Int or pVAX1 and equal amount of donor plasmids. Luciferase activity was measured at different time points. The data are summarized in Figures 7B (MLE12) and 7C (A549). With the three cellular promoters, the post integrative gene silencing as observed with pEGFPLucattB and pCCMVI-luc-attB was not 
A

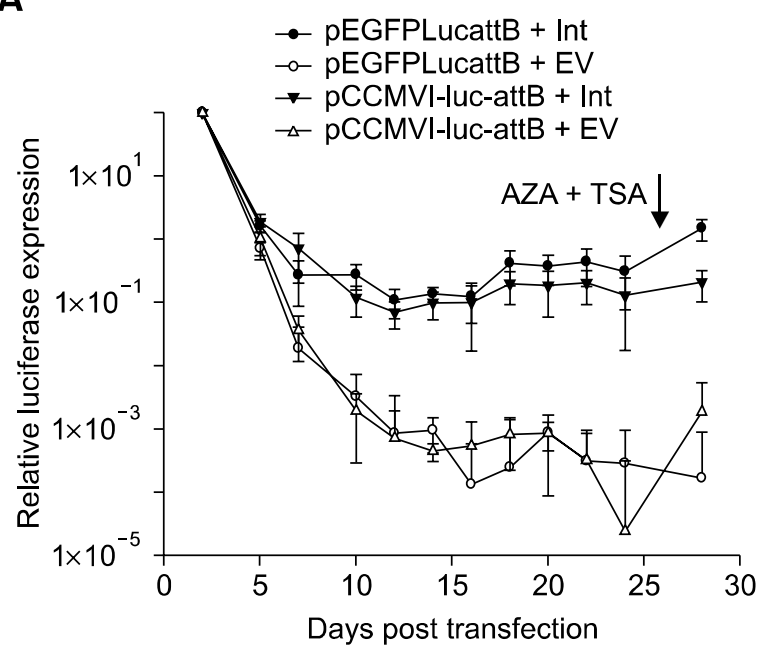

B

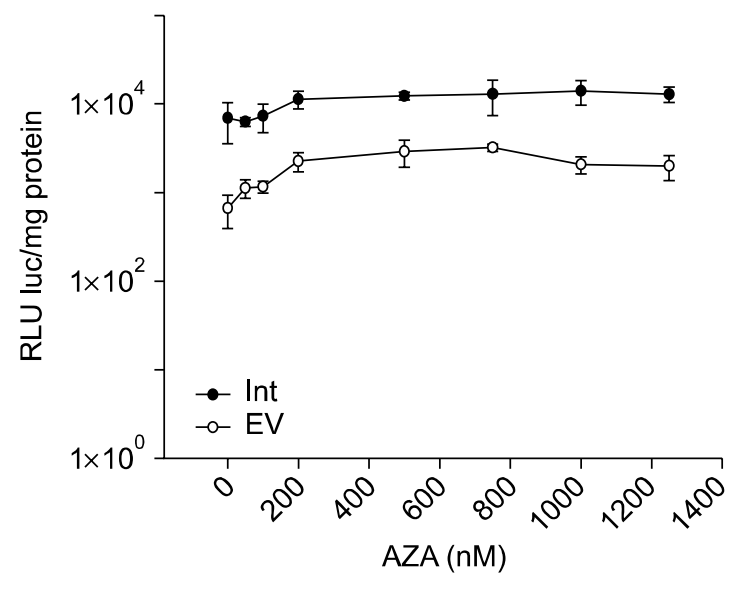

C

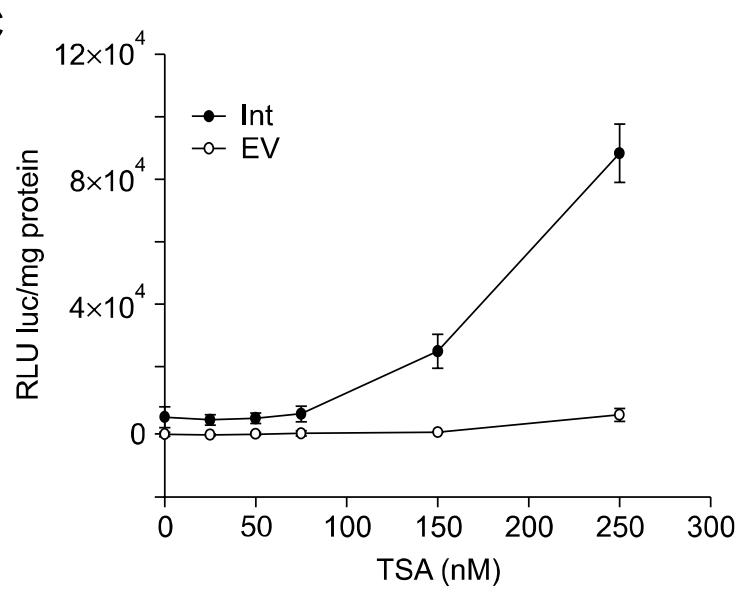

Figure 6. No effect of bacterial backbone on the long-term expression values observed in A549 cells after $\phi C 31$ integrase mediated integration. Constructs used have been schematically presented as Figure 5A. (A) A549 cells were transfected either with pEGFPLucattB \pm pVAX1-Int or pCCMVI-luc-attB \pm pVAX1-Int. AZA and TSA were added at 26 days post transfection. Luciferase activity was measured at different time points. Long-term expression results are expressed as percentage of luciferase values two days after transfection. (B) A549 cells were transfected with pEGFPLucattB \pm pVAX1-Int. Increasing concentrations of AZA were added at 26 days post transfection and luciferase measurements made $48 \mathrm{~h}$ subsequently. (C) A549 cells were transfected with pEGFPLucattB \pm pVAX1-Int. Increasing concentrations of TSA were added at 26 days post transfection and luciferase measurements made $48 \mathrm{~h}$ subsequently. Each transfection was repeated twice with two independent replicates per experiment. Values represent mean \pm standard deviation $(n=4)$. Int: Integrase construct; EV: pVAX1 used as vector control.

evident. Moreover, addition of AZA and TSA (21 days post transfection in MLE 12 cells and 26 days post transfection in A549 cells), had no significant effect on the expression values measured $48 \mathrm{~h}$ later. In the MLE12 cells there was no significant difference in the three cellular promoters. In A549 cells, EF1 $\alpha$ promoter resulted in significantly higher long-term expression values compared to the other two promoters $(P \leq 0.001)$.

To investigate if the observations made in A549 and MLE12 cells with respect to the effect of promoter and/or plasmid backbone were cell specific or a general phenomenon for lung cells, human bronchial epithelial cells (BEAS-2B) were transfected with each of the donor plasmid \pm pVAX1-Int/pVAX1. Luciferase based donor plasmids with the CMV promoter have been described as Figure $5 \mathrm{~A}$ and with cellular promoters been presented as Figure 7A. In contrast to the silencing of CMV promoter in alveolar epithelial cells, no silencing of this promoter could be observed in BEAS-2B (Figure 8A). Moreover, unlike in MLE12 cells where an improved plasmid backbone resulted in less extensive silencing, there was no effect of plasmid backbone on the resulting long-term expression (pEGFPLucattB and pCCMVI-luc-attB). Long-term expression values with donor plasmids based on cellular promoters, were in accordance 
A

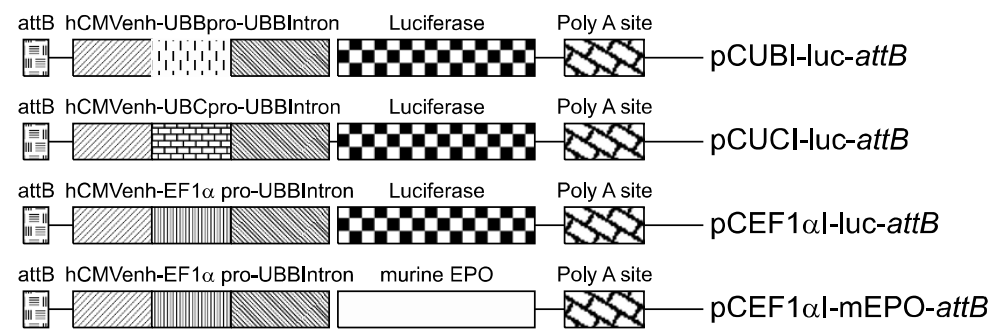

B

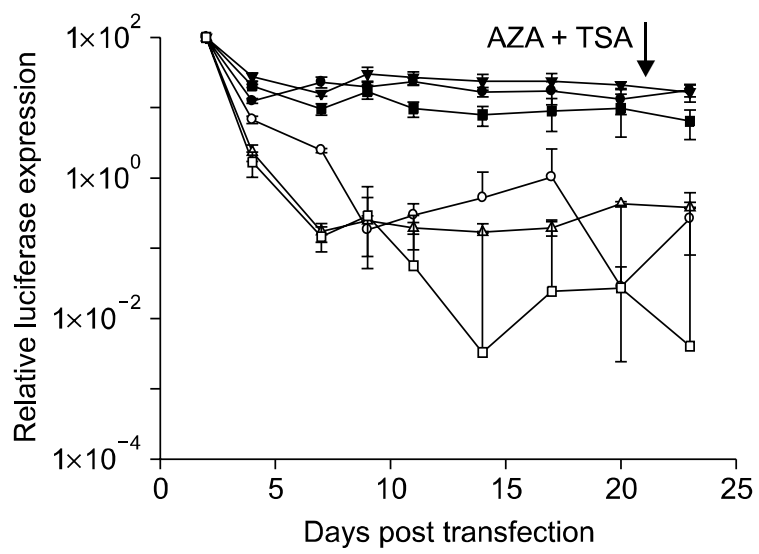

C

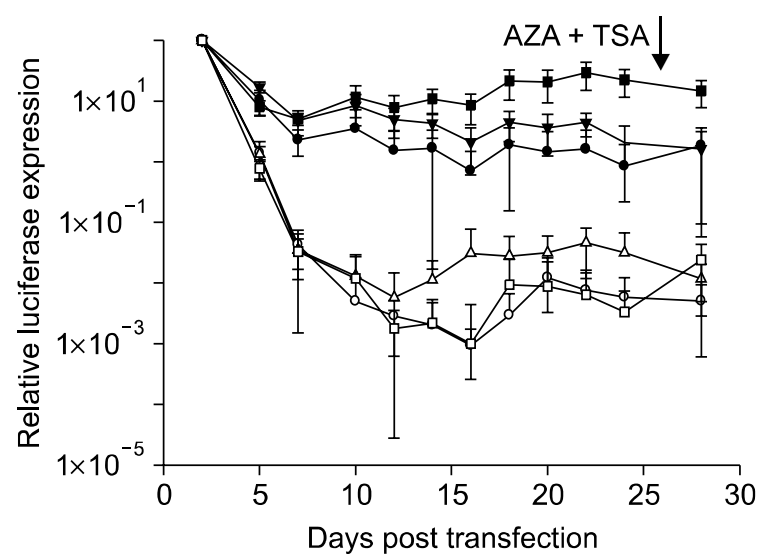

Figure 7. (A) Schematic representation of donor plasmids with cellular promoters used in this study. Cellular promoters are resistant to DNA methylase and HDAC mediated silencing in MLE12 (B) and A549 (C) cells. Cells were transfected with each of the different donor plasmids \pm pVAX1-Int. AZA and TSA were added at 21 days (MLE12) or 26 days (A549) post transfection. Luciferase activity was measured at different time points. Long-term expression results are expressed as percentage of luciferase values two days after transfection. Each transfection was repeated twice with two independent replicates per experiment.Values represent mean \pm standard deviation $(n=4)$. Int: Integrase construct; EV: pVAX1 used as vector control.

to the observations made in alveolar epithelial cells (Figure 8B), indicating their resistance to post-integrative gene silencing when integrated with $\phi \mathrm{C} 31$ integrase. In BEAS-2B, expression from UBC promoter resulted in significantly higher long-term expression values compared to the other two promoters $(P \leq 0.01)$

Since the donor plasmid with EF1 $\alpha$ promoter gave the desired results in A549 and MLE12 cells, the efficiency of this plasmid to support long-term expression when combined with $\phi C 31$ integrase, was checked in vivo in mice. Murine erythropoietin expression plasmid pCEF1al-mEPO-attB (Figure $7 A)$ was co-delivered with either pVAX1-Int or pVAX1. Luciferase expression plasmid (pCpG-luc) was co-delivered for both the groups to normalize for in vivo transfection efficiences. No significant difference could be observed between the PEI-pDNA complexes of both the groups (vector control vs. integrase) when compared with respect to their particle size and zeta potential (data not shown). Measurement of luciferase values by in vivo bioluminescent imaging (Aneja et al., 2007) at $24 \mathrm{~h}$ post IV injection showed similar levels of lung transfection in both the groups (data not shown).

Hematocrit values were measured for the blood samples collected at different time points post injection (Figure 9A). At day 30 post injection, hematocrit values of both the vector control and integrase groups were significantly higher compared to untreated group $(P<0.01)$. At subsequent time points, hematocrit values of the mice in the vector control group fell down to levels comparable to the untreated mice, whereas in the presence of integrase, hematocrit values were significantly higher $(P<0.01)$ till the end of the experiment (day 71). At 90 days post gene delivery, mice were euthanized and erythropoietin was quantified in the serum and homogenized lung lysates (Figure 9B). In the serum, erythropoietin levels in the integrase 
A

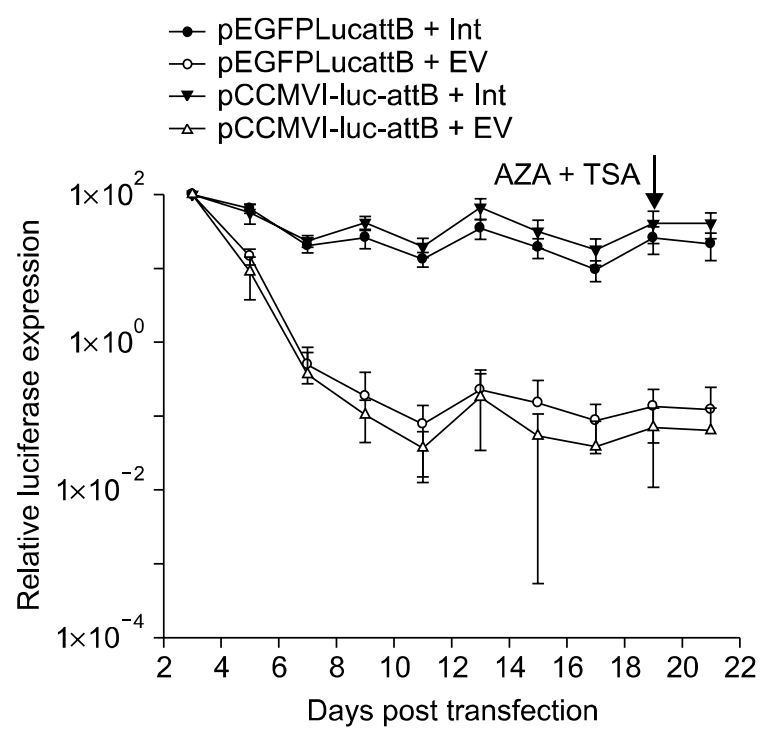

B

pCUBI-luc-attB + Int

$-a$ pCUBI-luc-attB + EV

$\rightarrow$ pCUCl-luc-attB + Int

$\triangle$ pCUCl-luc-attB + EV

$\rightarrow$ pCEF1 $\alpha$ l-luc-attB + Int

$\rightarrow$ pCEF1 $\alpha$ l-luc-attB + EV

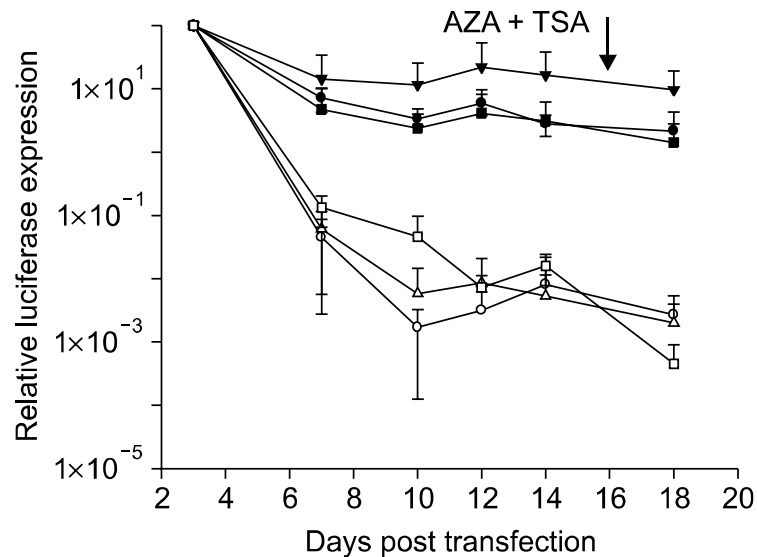

Figure 8. Effect of CMV promoter and bacterial backbone on long-term expression in BEAS-2B cells. (A) BEAS-2B cells were transfected either with pEGFPLucattB $\pm p V A X 1$-Int or pCCMVI-luc-attB $\pm p V A X 1$-Int. These constructs have been schematically presented as Figure $5 A$. (B) Cells were transfected with each of the different donor plasmids \pm pVAX1-Int. Constructs used have been schematically presented as Figure 7A. Luciferase activity was measured at different time points. AZA and TSA were added at 20 and 16 days post transfection ( $A$ and $B$ respectively). Long-term expression results are expressed as percentage of luciferase values three days after transfection. Each transfection was repeated twice with two independent replicates per experiment. Values represent mean \pm standard deviation $(n=4)$. Int: Integrase construct; EV: pVAX1 used as vector control.

group were observed to be $\sim 2$ fold higher $(P<$ $0.01)$ compared to the untreated or vector control mice. Similar trend was also observed in the lung lysates $(P<0.01)$. There was no difference in the serum erythropoietin levels between the untreated and the vector control groups at this time point in both serum and lung lysates.

\section{Discussion}

$\phi$ C31 integrase has been shown to mediate efficient genomic integration of pDNA into cells through a recombination event between the attachment sequence attB and pseudoattP sites present in the host genome (Thyagarajan et al., 2001). This type of recombination event, by requiring significant sequence identity, limits the number of potential integration sites, making the $\phi C 31$ integrase system relatively site-specific. In a study by Chalberg et al. (2006), it could be shown that $60 \%$ of the integrations occurred at only nineteen pseudo attP sites in three human cell lines. It has been proposed that even fewer sites may be used in vivo probably due to chromosomal context effects (Olivares et al., 2002). Moreover, the majority of the integration events occur once per cell (Chalberg et al., 2006). In addition, the recombination is unidirectional and integration is stable. These unique characteristics of the $\phi$ C31 integrase system make it an attractive nonviral integration system. This enzyme has been shown to mediate long-term expression of the transgene in different tissues like murine liver (Olivares et al., 2002), human keratinocytes (Ortiz-Urda et al., 2002) and muscle (Bertoni et al., 2006). We have recently shown the acitivity of the enzyme in lungs cells in vitro and in vivo (Aneja et al., 2007). However, the final levels of luciferase expression observed, appeared too low to be of physiological relevance with a therapeutic gene. The present study aimed to optimize the integrase system so as to reduce or prevent the steady decline in expression observed with the $\phi C 31$ integrase.

Our previous results in Jurkat cells with the integrase mRNA (Maucksch et al., 2008) and additional unpublished data led to the supposition that kinetics of integrase expression may influence its activity and the subsequent long-term expression observed. In this context, we next investigated if the choice of promoter or polyA site influenced the integrase activity. Three different promoters and two different polyA sites were compared with respect to their effects on the activity of $\phi C 31$ integrase using an intramolecular recombination assay. We have successfully used this recombination assay to detect integrase activity in our previous studies (Aneja et al., 2007; Maucksch et 
A

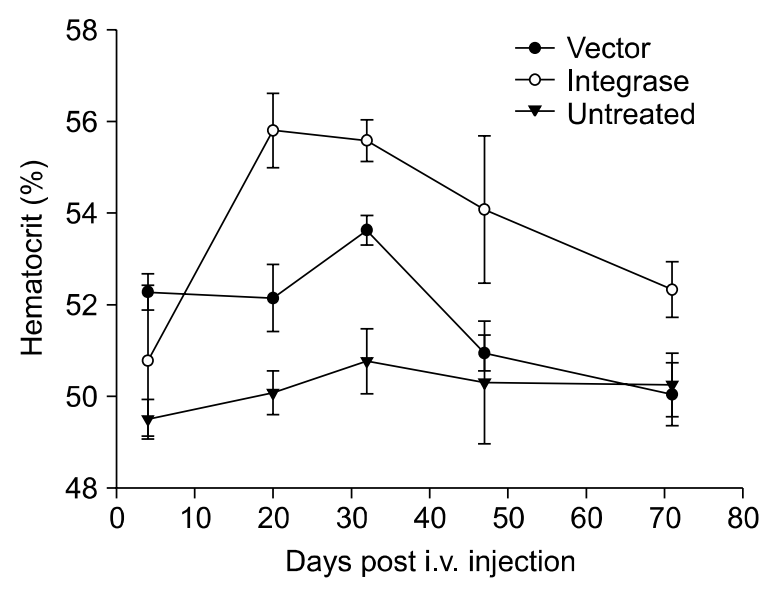

B

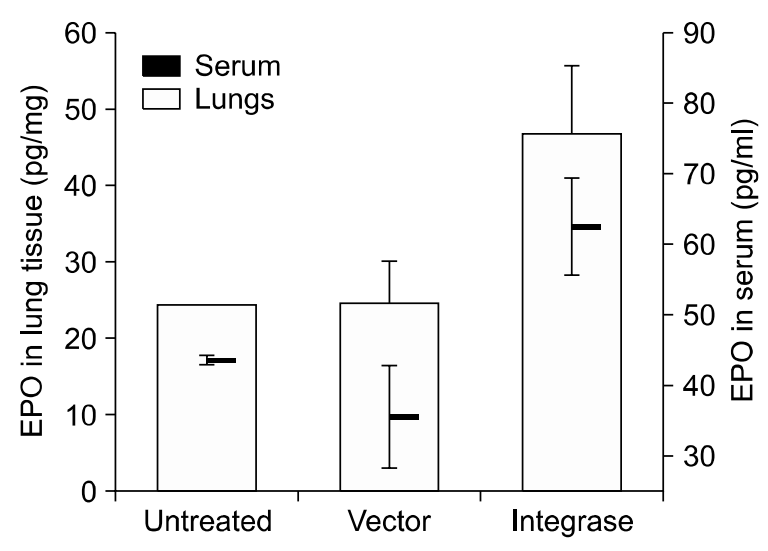

Figure 9. EF1 $\alpha$ promoter supports long-term physiological effects when combined with $\phi C 31$ integrase. Erythropoietin plasmid (pCEF1 $\alpha$ l-mEPO-attB: Figure 7A) was co-delivered with pVAX1-Int / pVAX1 via the intravenous route into mice ( $n=4$ per group). (A) Blood hematocrit values were measured at subsequent time points post gene delivery. Untreated mice were included as reference at every time point. (B) Erythropoietin levels were measured in lung lysates and serum by ELISA at three months post I.V. injection. Values represent mean \pm standard deviation. Vector: pVAX1 group; Integrase: pVAX1-Int group.

al., 2008). Using this assay, in A549 cells, CMV promoter and $\mathrm{BGH}$ polyA site resulted in significantly enhanced integrase activity compared to other promoters or SV40 polyA site respectively. In murine MLE12 cells however, while there was no effect of polyA site on the integrase activity, both CAG and CMV promoters resulted in higher integrase activity compared to PGK promoter. Results obtained in the intra-molecular recombination assay were substantiated by the long-term expression data comparing the effect of different parameters on $\phi \mathrm{C} 31$ integrase mediated long-term expression. These results provide evidence that the design of the integrase expression construct influences its activity in alveolar cells.

Unless $\phi C 31$ integrase contains an NLS, the 69 $\mathrm{kDa}$ protein is expected to be localized in the cytoplasm, because nuclear pore complexes inhibit the nuclear entry of proteins over about $40 \mathrm{kDa}$ (Dingwall and Laskey, 1986). To enhance the nuclear entry of this enzyme, NLS of the SV40 large $\mathrm{T}$ antigen, has been added at both the $\mathrm{N}$ and C-terminus of $\phi \mathrm{C} 31$ integrase (Andreas et al., 2002; Raymond and Soriano, 2007). Using an extrachromosomal plasmid carrying an attB and attP sites that must be recombined to express the reporter protein, it was reported that the $\phi \mathrm{C} 31$ integrase carrying a C-terminal NLS performed intramolecular excision 1.7-fold better than the wild-type enzyme (Andreas et al., 2002). When the same reporter plasmid was integrated into the chromosomes of NIH3T3 cells, expression of the reporter protein was 10 -fold higher in cells that received the $\phi C 31$ integrase with NLS compared to the wildtype (Andreas et al., 2002). However, for integration of plasmid DNA into the genome, the reaction of interest in gene therapy, $\phi C 31$ integrase with NLS has not been compared to the wild-type $\phi$ C31 integrase in either tissue culture cells or in a gene therapy setting. In the present study, we directly compared the integration of donor plasmid and subsequent long-term expression, when expression plasmids coding for an integrase with or without a C-terminal NLS were co-delivered. In our experiments, no beneficial effect of an NLS addition on integrase mediated long-term expression or the absolute amount of donor plasmid integration, could be observed in any of the investigated cell types. From the results it appears that genomic integration and subsequent long-term expression of the donor plasmid DNA is not limited by the amount of integrase protein in the nucleus. A small but significant amount of integrase protein may gain entry into the cell nucleus and this amount may be sufficient to achieve integration.

The second element of the integrase system is the donor plasmid which is to be integrated in the genome. Plasmid silencing has been implicated as a major cause of loss of transgene expression in eukaryotic cells (Chen et al., 2004). The cause for such loss of transgene expression has been attributed to the presence of $\mathrm{CpG}$ motifs and bacterial backbone sequences present in plasmid DNA. Recent studies have shown that depletion of CpG sequences in plasmid vectors can reduce the silencing effects and prolong gene expression (Yew et al., 2002; Chen et al., 2003). Moreover, the choice of promoter has also been shown to 
dramatically influence the longevity of expression (Gill et al., 2001; Yew et al., 2001). It has been proposed that transgenes integrated with $\phi \mathrm{C} 31$ integrase are also better expressed and not subjected to extensive silencing. The pseudoattP sites which are preferentially used by the integrase, are in the euchromatin (Olivares et al., 2002) as the DNA there is not so tightly packaged as in the heterochromatin. In this context, we obtained mixed results. When DNA methylase and HDAC inhibitors (AZA and TSA respectively) were added to MLE12 cells transfected with pEGFPLucattB, a significant 50 -fold increase was observed in the luciferase expression measured $48 \mathrm{~h}$ subsequently in both integrase and the control groups thus negating the beneficial effect of integration at pseudoattP in euchromatin to prevent gene silencing. Using these inhibitors separately provided evidence that both DNA methylases and HDACs are involved in gene silencing.

To separately investigate the contribution of bacterial backbone and promoter in the observed gene silencing, a previously described backbone pGZCUBI was used to construct new donor plasmids. This backbone contains an unmodified UBB promoter and intron, but a CpG reduced backbone composed of a synthetic, non-CpG gene conferring resistance to kanamycin and a minimal replication origin (Yew et al., 2001). Long-term expression experiments in MLE12 cells with pCCMVI-luc-attB revealed reduced silencing of the transgene when integrated with $\phi \mathrm{C} 31$ integrase compared to the control group. Thus, reducing the $\mathrm{CpG}$ motifs in the bacterial backbone did significantly reduce the post integrative gene silencing in murine cells.

However, in A549 cells, no effect of plasmid backbone was observed and expression from both pEGFPLucattB and pCCMVI-luc-attB declined in a similar manner, thus indicating that reducing the CpG motifs in the bacterial backbone did not result in any significant improvement in the longevity of expression. This could be due to the fact that DNA methylases may not be the key mediators of post-integrative gene silencing in A549 cells, as reflected by the lack of increase in luciferase expression subsequent to the treatment of cells with $A Z A$ alone. Besides, these differences with respect to the role of bacterial backbone in post integrative gene silencing in MLE12 and A549 cells could also be due to other species specific differences between the two cell types. Nonetheless, these results provided the evidence that the donor plasmid was silenced in a post-integrative manner. Similar post-integration gene silencing has also been observed for the Sleeping Beauty transposon system (Garrison et al., 2007). In contrast to the silencing of the CMV promoter in alveolar epithelial cells, stable long-term expression was achieved in BEAS-2B cells when donor plasmids were integrated with $\phi C 31$ integrase. Addition of AZA and TSA had no effect of expression measured $48 \mathrm{~h}$ subsequently, providing evidence for the lack of post-integrative silencing. These results in different lung cells suggest that the expression/silencing of CMV promoter may be cell type specific.

In order to design better donor plasmids, CMV promoter was replaced by cellular promoters namely UBB, UBC and EF1 $\alpha$. In previous studies, long-term expression from plasmid vectors has been achieved using a hybrid UBB (Yew et al., 2001), UBC and EF1 $\alpha$ promoters (Gill et al., 2001). We here attempted to combine the desirable features of cellular promoters with the integration activity of $\phi$ C31 integrase. Though relatively long-term expression with plasmids containing these promoters has been reported (Gill et al., 2001; Yew et al., 2001), nothing is known about the kinetics and duration of expression when constructs bearing these promoters are integrated with $\phi C 31$ integrase. Since integration with $\phi C 31$ integrase happens at specific sites in the genome (pseudoattP), it may place these promoters in a different chromatin context when compared to the unintegrated plasmid. In the absence of integrase, there was a steady decline in expression from the donor plasmids containing these cellular promoters, most probably due to the loss of plasmid during cell division. However, when integrated by $\phi$ C31 integrase, each of the cellular promoters supported steady long-term expression. Cell type specific differences were also observed between the resulting long-term expression values observed with the different cellular promoters. Whereas in MLE12 cells, there was no significant difference between the compared cellular promoters, in human A549 cells, EF1 $\alpha$ promoter supported significantly higher long-term expression values compared to the other two promoters. In BEAS-2B cells, expression from UBC promoter was significantly higher compared to UBB and EF1 $\alpha$ promoters. Addition of AZA and TSA clearly revealed that these improved donor plasmids were resistant to post integrative gene silencing.

In situ production of a secreted therapeutic protein is one of the major gene therapy applications. Lungs are attractive targets for such applications as the entire blood volume flows through the lungs for exchange of respiratory gases. Auricchio et al. (2002) using AAV vectors and erythropoietin as a physiological marker gene, have shown the feasibility of non invasive gene transfer to the lungs for systemic delivery of therapeutic proteins. Our 
results with the $\phi \mathrm{C} 31$ integrase provide an alternative to viral vectors for the production of erythropoietin from the lungs. Long-term expression of murine erythropoietin, as measured by ELISA, could be observed in the presence of integrase. Moreover, the amounts produced in vivo resulted in a physiological response as measured by significant increase in the blood hematocrit values.

These results provide evidence that the choice of the donor plasmid is the critical factor of the $\phi C 31$ integrase system in achieving stable long-term expression. The donor plasmids need to be optimized based on the target tissue and the species as both tissue specific and species specific differences may influence the integrase activity and the subsequent long-term transgene expression. Tissue specific activity and specificity of $\phi \mathrm{C} 31$ integrase has been reported previously (Ehrhardt et al., 2006; Maucksch et al., 2008).

The results presented here, in addition to providing evidence for post integrative gene silencing with the $\phi C 31$ integrase system, also demonstrate that gene silencing mechanisms may differ across cell types and species. With this optimization, $\phi$ C 31 integrase presents itself as a promising nonviral gene therapy system for pulmonary disorders.

\section{Methods}

\section{Plasmids}

Integrase expression plasmid pCMVInt has been previously described (Thyagarajan et al., 2001; Olivares et al., 2002; Ortiz-Urda et al., 2002) and was kindly provided by MP Calos, Department of Genetics, Stanford University. pVAX1-Int was constructed by excising the integrase cDNA from pCMV-Int by Pstl-Xhol (MBI Fermentas) digestion and cloning into respective sites of pVAX1 (Invitrogen, Germany). pCAG-Int (NLS) and pPGK-Int (NLS) coding for the $\phi C 31$ integrase with a C-terminal SV40 T-antigen NLS and driven by CAG and PGK promoters, respectively, were kindly provided by Ralf Kuehn, Institute of Developmental Genetics, Helmholtz Centre, Neuherberg, Germany. $\phi C 31-N L S$ was excised from pCAG-Int (NLS) by Pacl-Sacl (New England Biolabs, Germany) and cloned into the EcoRI site of pVAX1 by blunt end ligation to generate pVAX1-Int (NLS). Integrase reporter plasmid pSVpaxattP50attB53, has been previously described (Aneja et al., 2007; Maucksch et al., 2008) and was kindly provided by Ralf Kuehn. pVAX1-luc was constructed by excising firefly luciferase cDNA from pGL3-Basic vector (Promega) by HindIII-Xbal digestion and cloning into respective sites of pVAX1. pVAX1-luc was used to normalize the transfection efficiencies of different samples.

Among donor plasmids, pEGFPLucattB was kindly provided by MP Calos and has been described previously (Aneja et al., 2007). pGZCUBI plasmid backbone has been described previously (Yew et al., 2001) and was kindly provided by SH Cheng (Genzyme Corporation). pCUBI-luc was constructed by excising luciferase from pVAX1-luc by HindlII-Pmel (New England Biolabs, Germany) and cloning into the Notl (Fermentas, Germany) site of pGZCUBI. $\phi$ C31 integrase recognition sequence attB was excised from pTA-attB (provided by MP Calos) by EcoRI (Fermentas, Germany) digestion and was cloned blunt ended into the Kpnl (Fermentas, Germany) site of pCUBI-luc to obtain pCUBI-luc-attB. Ubiquitin C promoter (UBC) was excised from pUB6/V5-His/lacZ (Invitrogen, Germany) by Bg/ll-HindIII (Fermentas, Germany), EF1 $\alpha$ promoter (EF1 $\alpha$ ) from pWPXL (Addgene Inc. Cambridge) by Sall-Pacl (New England Biolabs, Germany) and cytomegalovirus immediate early promoter (CMV) from pEGFPLucattB by EcoRI-Nhel (Fermentas, Germany) digestions respectively. Ubiquitin B (UBB) promoter was excised from pCUBI-luc-attB by BamHI-Avrll (Fermentas, Germany) digestion and replaced by UBC, EF1 $\alpha$ or CMV promoters to generate pCUCl-luc-attB, pCEF1 $1 \alpha$ l-luc-attB and pCCMVI-luc-attB respectively. Murine erythropoietin cDNA was excised from pCR4-TOPO-mEPO (Open Biosystems) by EcoRI and cloned blunt ended into pCEF1 $\alpha$ l-luc-attB by replacing luciferase (Ncol-Sfil) to generate pCEF1 $\alpha$ mEPO-attB.

\section{Cell culture and transfections}

Murine and human alveolar type II epithelial cell lines (MLE12 and A549, respectively) were obtained from German Collection of Microorganisms and Cell Cultures. Human bronchial epithelial cells (BEAS-2B) were obtained from American Type Culture Collection. All cells were grown in Minimal Essential Medium (Gibco-BRL, Karlsruhe, Germany) supplemented with $10 \%$ FCS, $1 \%$ penicillin-streptomycin and $0.5 \%$ gentamycin.

One day prior to transfection, 80,000 cells/well were plated in 24 well plates. Lipofectamine 2000 was used as a transfection reagent and complexes were prepared following manufacturer's instructions. For intramolecular recombination assay, cells were transfected with a DNA mix consisting of $100 \mathrm{ng}$ of pSVpaxattP50-attB53+50 $\mathrm{ng}$ of pVAX1-luc and equal number of molecules of the different integrase encoding plasmids. pVAX1 was used as vector control and also filler DNA to make the total amount of DNA (800 ng) equal in all the compared samples. For long-term expression studies, cells were transfected with $200 \mathrm{ng}$ of donor plasmid together with equal number of molecules of the different integrase encoding plasmids. pVAX1 was used as filler DNA to make the total amount of DNA (800 ng) equal in all the compared samples. At day 1 post transfection, plates for long-term expression were split $1: 2$ and incubation continued for another $24 \mathrm{~h}$. One of the plates was used for luciferase measurement and the other plate was incubated and split regularly (2-3 days) to obtain cells at different time points.

\section{Reporter gene assays}

Luciferase and $\beta$-galactosidase assays were performed at different time points post transfection. Prior to lysis for luciferase/ $\beta$-galactosidase measurement, cells were washed twice with PBS followed by the addition of $100 \mu$ of lysis 
buffer (25 mM Tris- $\mathrm{HCl}, 0.1 \%$ Triton $\mathrm{X}-100, \mathrm{pH}=7.8)$. Cells were incubated for $10 \mathrm{~min}$ at room temperature in the lysis buffer. $5 \mu \mathrm{l}$ of the lysate was used to quantify the protein amount with BioRad protein assay (BioRad, Munich, Germany) using a bovine serum albumin standard. For measurement of $\beta$-galactosidase activity, $\beta$-gal reporter gene assay kit (Roche Diagnostics $\mathrm{GmbH}$, Mannheim, Germany) was used following manufacturer's protocol. Luciferase assay was performed as described previously (Aneja et al., 2007; Maucksch et al., 2008). $50 \mu$ lof the cell lysate was used to measure luciferase/ $\beta$-galactosidase activity on a Wallac Victor ${ }^{2} 1420$ Multilabel Counter (Perkin Elmer, Rodgau-Jügesheim, Germany). Both luciferase/ $\beta$ galactosidase activity values were normalized with respect to the protein concentration. Results for intramolecular recombination are presented as mean \pm standard deviation of the ratio of values obtained for $\beta$-galactosidase and luciferase. Long-term expression results are expressed as percentage of luciferase values two or three days after transfection. Mann-Whitney test was used to compare the samples and determine statistical significance.

\section{DNA methylase and HDAC inhibitors}

DNA methylases inhibitor 5-aza-2'deoxycytidine (AZA) and histone deacetylase complex (HDAC) inhibitor trichostatin A (TSA) were used at the final concentrations of $1.25 \mu \mathrm{M}$ and $0.25 \mu \mathrm{M}$, respectively, or as indicated.

\section{Real time PCR}

Total DNA was isolated from MLE12 cells at 22 days post transfection, using the DNeasy Tissue Kit (Qiagen, Hilden, Germany) according to manufacturer's instructions. To detect and quantify pEGFPLucattB, following primer pair was used: FPLuc, 5'-TCAAAGAGGCGAACTGTGTG-3' and RPLuc, 5'-GGTGTTGGAGCAAGATGGAT-3'. Real time PCR was performed with $100 \mathrm{ng}$ of isolated DNA with a program of $10 \mathrm{~min}$ at $94^{\circ} \mathrm{C} ; 35$ cycles of $94^{\circ} \mathrm{C}$ for $30 \mathrm{~s}, 60^{\circ} \mathrm{C}$ for $20 \mathrm{~s}$ and $72^{\circ} \mathrm{C}$ for $30 \mathrm{~s}$; followed by $10 \mathrm{~min}$ at $72^{\circ} \mathrm{C}$ using $\mathrm{iQ}$ Supermix (BioRad, Hercules, CA). Standard curve of pEGFPLucattB target plasmid was generated to measure absolute amounts. Ct values were obtained using iCycler iQ software 3.1 (BioRad, Hercules, CA).

\section{In vivo experiments}

Six- to eight-week-old female BALB/c mice (Charles River Laboratories, Sulzfeld, Germany) were maintained under specific-pathogen-free conditions. Animals were acclimatized for at least 7 days prior to the start of the experiments. All animal procedures were approved and controlled by the local ethics committee and carried out according to the guidelines of the German law of protection of animal life. Plasmids used in in vivo experiments were amplified using the mega prep kit from Macherey-Nagel (Dueren, Germany).

\section{Intravenous injection of the complexes into the tail vein and luciferase expression}

$20 \mu \mathrm{g}$ of pCEF1 $\alpha$-mEPO-attB plus $20 \mu \mathrm{g}$ of pVAX1-Int /
pVAX1 and $10 \mu \mathrm{g}$ pCpG-luc (a luciferase expression plasmid) and L-PEI $22 \mathrm{kDa}$ (kindly provided by Manfred Ogris and Ernst Wagner, Department of Pharmacy, LMU, Munich) were diluted separately in $200 \mu$ of $5 \%$ glucose. Amount of L-PEI was calculated at N/P ratio of 10 . The DNA solution was pipetted into the PEI solution, homogenised immediately by pipetting up and down and incubated for $10 \mathrm{~min}$ at room temperature. $400 \mu \mathrm{l}$ of PEI-DNA polyplexes/mouse ( $50 \mu \mathrm{g}$ of DNA per mouse) were injected into the tail vein within $30 \mathrm{~s}$, using a $1 / 2$ inch 26-gauge needle and a $1 \mathrm{ml}$ syringe.

\section{Hematocrit and ELISA measurements}

Blood was collected from the mice via the retro-orbital route and hematocrit values were measured on Automatic Hemoanalyser (Sysmex, Germany). At 3 months post gene delivery, mice were euthanized and lungs and blood were isolated. Serum prepared from the blood and lungs were homogenised and lysed in the buffer $(25 \mathrm{mM}$ Tris- $\mathrm{HCl}$, $0.1 \%$ Triton $\mathrm{X}-100, \mathrm{pH}=7.8$ ) supplemented with Complete Protease Inhibitor Cocktail ${ }^{\mathrm{TM}}$ (Roche, Germany). Erythropoietin amounts were quantified in the prepared serum and homogenised lung lysates by ELISA (R\&D Systems, Abingdon, UK). Results for hematocrit and erythropoietin measurements are mean \pm standard deviation.

\section{Acknowledgements}

The authors would like to thank Michele Calos (Stanford University) for providing pEGFPLucattB, pCMVInt and pTA-attB. The generous gift of the following plasmids: pCAG-Int (NLS), pPGK-Int (NLS) and pSVpaxattP50-attB53 by Ralf Kuehn (Helmholtz Center, Munich, Germany) is highly appreciated. We would also like to thank Corinna Pfeifer for her assistance with statistics. This work was supported by the German Federal Ministry of Education and Research in the program BioFuture (0311898), 01GU0616, Magselectofection (LSHB-CT-2006-019038) and LMUexcellent (Investitionsfonds).

\section{References}

Andreas S, Schwenk F, Kuter-Luks B, Faust N and Kuhn R. Enhanced efficiency through nuclear localization signal fusion on phage PhiC31-integrase: activity comparison with Cre and FLPe recombinase in mammalian cells. Nucleic Acids Res 2002;30:2299-306

Aneja MK, Imker R and Rudolph C. Phage phiC31 integrase-mediated genomic integration and long-term gene expression in the lung after nonviral gene delivery. J Gene Med 2007;9:967-75

Auricchio A, O'Connor E, Weiner D, Gao GP, Hildinger M, Wang L, Calcedo R and Wilson JM. Noninvasive gene transfer to the lung for systemic delivery of therapeutic proteins. J Clin Invest 2002;110:499-504

Bertoni C, Jarrahian S, Wheeler TM, Li Y, Olivares EC, Calos MP and Rando TA. Enhancement of plasmid-mediated gene therapy for muscular dystrophy by directed plasmid inte- 
gration. Proc Natl Acad Sci USA 2006;103:419-24

Chalberg TW, Portlock JL, Olivares EC, Thyagarajan B, Kirby PJ, Hillman RT, Hoelters $\mathrm{J}$ and Calos MP. Integration specificity of phage phiC31 integrase in the human genome. J Mol Biol 2006;357:28-48

Chen ZY, He CY, Ehrhardt A and Kay MA. Minicircle DNA vectors devoid of bacterial DNA result in persistent and high-level transgene expression in vivo. Mol Ther 2003; 8:495-500

Chen ZY, He CY, Meuse L and Kay MA. Silencing of episomal transgene expression by plasmid bacterial DNA elements in vivo. Gene Ther 2004;11:856-64

Davis PB and Cooper MJ. Vectors for airway gene delivery. AAPS J 2007;9:E11-7

Demeneix B and Behr JP. Polyethylenimine (PEI). Adv Genet 2005;53:217-30

Dif F, Djediat C, Alegria O, Demeneix B and Levi G. Transfection of multiple pulmonary cell types following intravenous injection of PEI-DNA in normal and CFTR mutant mice. J Gene Med 2006;8:82-9

Dingwall $C$ and Laskey RA. Protein import into the cell nucleus. Annu Rev Cell Biol 1986;2:367-90

Ehrhardt A, Engler JA, Xu H, Cherry AM and Kay MA. Molecular analysis of chromosomal rearrangements in mammalian cells after phiC31-mediated integration. Hum Gene Ther 2006;17:1077-94

Garrison BS, Yant SR, Mikkelsen JG and Kay MA. Postintegrative gene silencing within the Sleeping Beauty transposition system. Mol Cell Biol 2007;27:8824-33

Gill DR, Smyth SE, Goddard CA, Pringle IA, Higgins CF, Colledge WH and Hyde SC. Increased persistence of lung gene expression using plasmids containing the ubiquitin $\mathrm{C}$ or elongation factor 1alpha promoter. Gene Ther 2001;8: 1539-46

Goula D, Becker N, Lemkine GF, Normandie P, Rodrigues J,
Mantero S, Levi G and Demeneix BA. Rapid crossing of the pulmonary endothelial barrier by polyethylenimine/DNA complexes. Gene Ther 2000;7:499-504

Held PK, Olivares EC, Aguilar CP, Finegold M, Calos MP and Grompe M. In vivo correction of murine hereditary tyrosinemia type I by phiC31 integrase-mediated gene delivery. Mol Ther 2005;11:399-408

Lee TWR, Matthews DA and Blair GE. Novel molecular approaches to cystic fibrosis gene therapy. Biochem J 2005;387:1-15

Maucksch C, Aneja MK, Hennen E, Bohla A, Hoffmann F, Elfinger $\mathrm{M}$, Rosenecker $\mathrm{J}$ and Rudolph $\mathrm{C}$. Cell type differences in activity of the Streptomyces bacteriophage phiC31 integrase. Nucleic Acids Res 2008;36:5462-71

Olivares EC, Hollis RP, Chalberg TW, Meuse L, Kay MA and Calos MP. Site-specific genomic integration produces therapeutic Factor IX levels in mice. Nat Biotechnol 2002;20:1124-8

Ortiz-Urda S, Thyagarajan B, Keene DR, Lin Q, Fang M, Calos MP and Khavari PA. Stable nonviral genetic correction of inherited human skin disease. Nat Med 2002;8:1166-70

Raymond CS and Soriano P. High-efficiency FLP and PhiC31 site-specific recombination in mammalian cells. PLoS ONE 2007;2:e162

Thyagarajan B, Olivares EC, Hollis RP, Ginsburg DS and Calos MP. Site-specific genomic integration in mammalian cells mediated by phage phiC31 integrase. Mol Cell Biol 2001;21:3926-34

Yew NS, Przybylska M, Ziegler RJ, Liu D and Cheng SH. High and sustained transgene expression in vivo from plasmid vectors containing a hybrid ubiquitin promoter. Mol Ther 2001;4:75-82

Yew NS, Zhao H, Przybylska M, Wu IH, Tousignant JD, Scheule RK and Cheng SH. CpG-depleted plasmid DNA vectors with enhanced safety and long-term gene expression in vivo. Mol Ther 2002;5:731-8 